\section{Comment on: A comparative study of online learning in response to the Coronavirus disease 2019 pandemic versus conventional learning.}

\section{To the Editor}

We have read with interest the study by Hanafy et al. ${ }^{1}$ Despite the development of 2019 novel coronavirus (COVID-19) vaccines and several treatments, COVID-19 is still a modern plague that threatens human health due to insufficient vaccination rates, vaccine inequity, and variants. ${ }^{2}$ Hanafy et $\mathrm{al}^{1}$ suggested in a recent study that it is desirable to provide both online and conventional learning methods, called blended learning, to maximize the benefits in undergraduate medical education considering the pros and cons of each in the midst of a global public health crisis, COVID-19 epidemic. This article on the relationship between the COVID-19 pandemic and medical education covers a very timely topic. In particular, the composition of the questions that grasped the important points of the current situation and the survey on the difference in preference for online or offline was very interesting to us who had experienced a similar situation. The COVID-19 pandemic situation has been confusing for both students and educators around the world. Similarly, South Korea did not properly prepare educationally for a long-term epidemic situation in the early stages of the COVID-19 epidemic. The urgently conducted recorded lectures and real-time online lectures did not conform to the classical scoring methods. However, a semester has passed by gradually setting new class strategies and scoring standards suitable for online lectures, and now, as suggested by Hanafy et $\mathrm{al},{ }^{1}$ we are implementing our own unique blended learning as follows. In our medical school, there are 126 students per academic year, of which 12 students in one classroom according to social distancing measures are given face-to-face classes. At the same time, an online class is provided to the rest of the students with video recorded in real time. In other words, students attend face-to-face classes when it is their turn, and the rest attend online classes. It seems that our attempts and the study by Hanafy et $\mathrm{al}^{1}$ can provide as educational resources for blended learning. This article by Hanafy et $\mathrm{al}^{1}$ has high value in that it is a study on the transition between online learning and conventional learning, which may never happen again, in a situation where there is no choice but to do online learning in many things. In addition, this study evaluated how the relatively old and unfamiliar educators of online classes accepted the online learning as well as most of the young learners who were likely to have adapted well to online learning. This helped to listen to various opinions about online learning, and it made it possible to understand the opinions of those who have difficulty adapting to online classes.

However, I would like to make a few comments about the study. First, the interpretation of the comparison of examination scores between the midterm and final examinations may have been biased. In addition to the difference between offline midterm examination and online final examination, the contents of the 2 examinations are different, and data on the characteristics of item, such as item difficulty and item discrimination between the 2 examinations, are not presented in this study. ${ }^{1}$ In addition, according to the survey on the time required for the examination, it is assumed that the time limit for the online/offline examination is different, which makes it difficult to guarantee the consistency of the examination situation. Rather, it would be a good comparison to compare the examination results of this year's online teaching with the same content as the examination results of last year's conventional teaching. In another previous study, ${ }^{3}$ when comparing academic achievement between conventional and online teaching, the results of the online teaching were compared with the students' scores in the previous year's conventional teaching. Except for the differences in education delivery methods, that is, online or offline mode, teaching professors, textbooks, class contents, and examination scope were all the same. In addition, even if the online teaching was implemented, the examination was conducted in offline mode. Second, by asking students about the risks of cheating and fraud, Hanafy et $\mathrm{al}^{1}$ can only know the overall perception of students on examination cheating, but it is difficult to accurately determine the actual cheating rate. In a previous study on cheating detection, a network internet protocol detector and the behavior detector were used to detect cheating in an online examination. ${ }^{4}$ It is also possible to introduce a system that technically prevents cheating in advance. ${ }^{5}$ Finally, regarding the selection of study subjects, the subjects of this study were students who were urgently switched to online education due to the COVID-19 pandemic. Of course, this was a good study to resolve the question regarding the effectiveness of online education that many educational institutions around the world have. However, the online education experience in the first and second semesters after COVID-19 pandemic can be completely different. Satisfaction and examination 
results immediately after starting online education in the first semester may be different from those in the second semester after experiencing and adjusting to online education for one semester. To better compare online teaching and conventional teaching, it would be better to compare the conventional education experience with the data after adapting to online teaching, even if there is one-year gap. It would be a great help for better understanding this study if the authors provide us some comments and explanations regarding several of our questions so far.

Sang Yeoup Lee Department of Medical Education Pusan National University School of Medicine Yangsan, Korea

Min Gi Shin Department of Medicine Pusan National University School of Medicine Yangsan, Korea

Si Gyoung Yu Department of Medicine Pusan National University School of Medicine Yangsan, Korea
Da Woon Jung

Department of Medicine Pusan National University School of Medicine Yangsan, Korea

Saudi Med J 2021; Vol. 42 (10): 1153-1154 doi: 10.15537/smj.2021.42.10.20210599

\section{Reply from the Author}

No reply was received from the Author.

\section{References}

1. Hanafy SM, Jumaa MI, Arafa MA. A comparative study of online learning in response to the coronavirus disease 2019 pandemic versus conventional learning. Saudi Med J 2021; 42 : 324-331.

2. Padma TV. COVID vaccines to reach poorest countries in 2023 - despite recent pledges. Nature 2021; 595: 342-343.

3. Cho SI. Comparison of academic achievement of classroom learning and on-line learning: a case study. J Bus Educ 2020; 34: 23-39.

4. Tiong LCO, Lee HJ. E-cheating prevention measures: detection of cheating at online examinations using deep learning approach - a case study. asXiv 2021: 2101.09841.

5. Li M, Luo L, Sikdar S, Nizam NI, Gao S, Shan H, Kruger M, Kruger U, Mohamed H, Xia L, Wang G. Optimized collusion prevention for online exams during social distancing. NPJ Sci Learn 2021; 6: 5. 